\title{
Dynamics of the Catalytic Active Site of Isoleucyl tRNA Synthetase from Staphylococcus Aureus bound with Adenylate and Mupirocin
}

Author: Shilpi Chowdhury, Nilashis Nandi*,1

Department of Chemistry, Kalyani University, Kalyani, West Bengal, India, 741235

\section{Supporting information}

S1. Reaction Scheme of two-step aminoacylation in IleRS

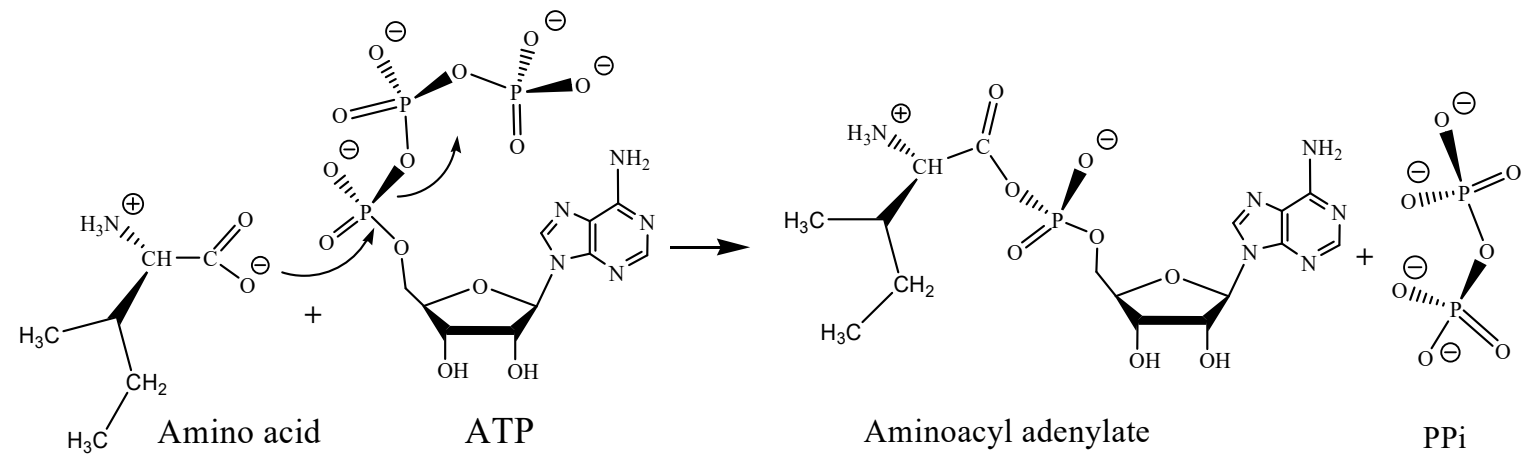

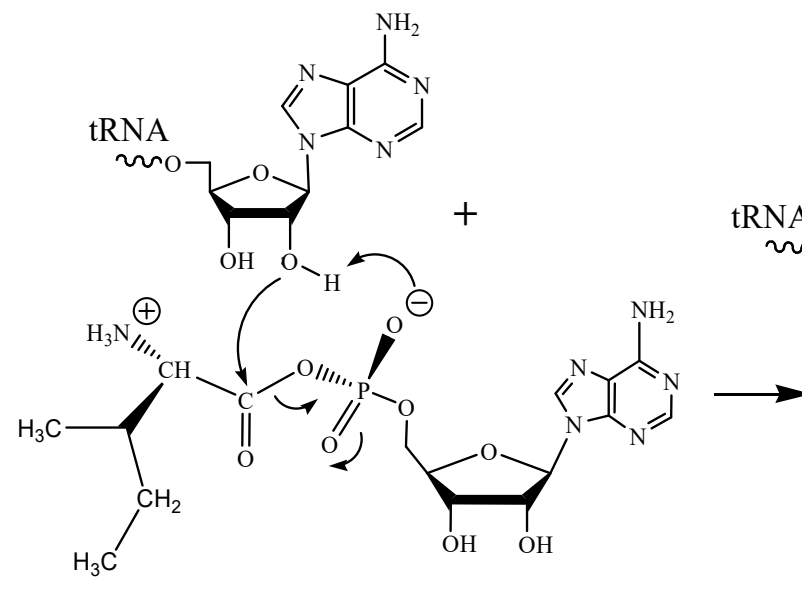

Aminoacyl adenylate

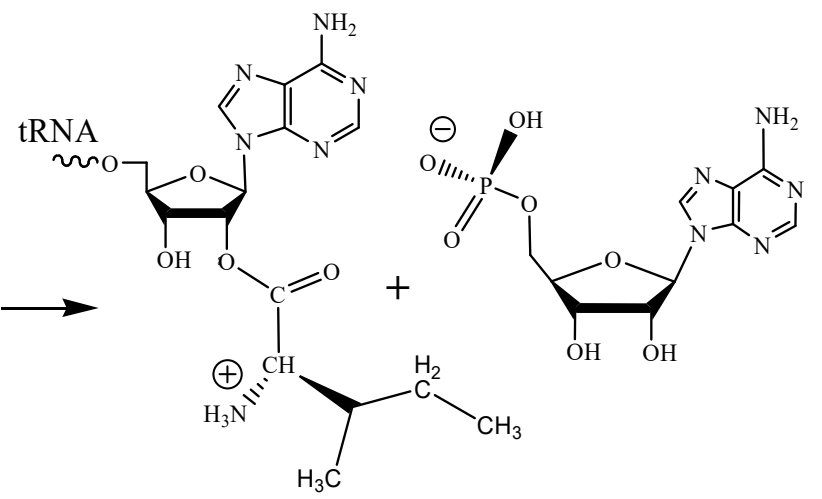

aa-tRNA

AMP

Fig. S1. The reaction scheme of formation of isoleucyl adenylate (adenylation step) and charging of tRNA with isoleucine is shown. Aminoacylation reaction is a two-step reaction. The cognate aa and adenosine triphosphate (ATP) react to form aminoacyl adenylate (aa-AMP or Ile-AMP in the present case) in the first step (the adenylation reaction). In the second step (the charging of amino acid over the acceptor stem of tRNA), the 2'- or 3'- hydroxyl group of the terminal adenosine of the tRNA attacks the $\alpha$-carboxylic carbon of adenylate leading to the formation of tRNA ${ }^{\text {aa }}$. The mupirocin is an analogue of isoleucyl adenylate (IleAMP). 
S2. Primary sequence of ${ }^{S a}$ IleRS

$\alpha 1$

Turn1

MDYEKTLLMPKTDFPMRGGLPNKEPQIQEKWDAED
Loop 1

$\alpha 2$

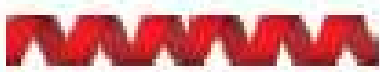

QYHKALEKNKGNETFILHDGPPYANGNL

Loop 2

$\alpha 3$

HMGHALNKILKDFIVRYKTMQGFYAPYVPGWDTH

Loop 3

$\alpha 4$

a5

GLPIEQALTKKGVDRKKMSTAEFREKCKEFALEQIEL

Loop 4

as

$\alpha 6$

QKKDFRRLGVRGDFNDPYITLKPEYEAAQIRIFGEMADKGL

Loop 5

Turn2

$\beta 1$

Turn 3

$\beta 2$

IYKGKKPVYWSPSSESSLAEAEIEYHDKRSASIYVAFNV 
YNVGEKYIIAEALSDAVAEALDWDKASIKLEKEYTGKKLEKE 287 Loop 7

YTGKELEWVVAQHPFLDRESLVINGDHVTTDAGTGCVHTAPG 329

B3 Turn $4 \quad \beta 4 \quad \alpha 8$

LDFITHSYPHDWRTKKPVIFRATPQWFASISKVRQDILDAIENT 430

Turn 5

$\alpha 9$

$\beta 5$

NFKVNWGKTRIYNMVRDRGEWVISRQRVWGVPLPVFYAE

$$
\alpha 10 \quad \text { Turn } 6 \quad \alpha 11
$$

\section{Loop 8}

NGEIIMTKETVNHVADLFAEHGSNIWFEREAKDLLPEGFTHPGS 513 Loop 9

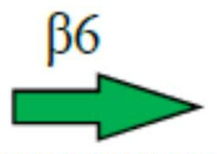

$\alpha 12$

$\beta 7$

PNGTFTKETDIMDVWFDSGSSHRGVLETRPELSFPADMYLEGS 556 Loop $10 \quad$ Loop 11 Loop 12 


\section{ArNace}

DQYRGWFNSSITTSVATRGVSPYKFLLSHGFVMDGEGK

Loop 13

$$
\text { Loop } 14
$$

$\alpha 14$

$$
\text { Turn } 7 \quad \alpha 15
$$

KMSKSLGNVIVPDQVVKQKGADIARLWVSSTDYLADVRIS

634

Loop 15

Loop 16

$\alpha 16$

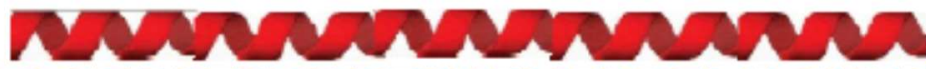

DEILKQTSDDYRKIRNTLRFMLGNINDFNPDTDSIPESEL

Loop 17

$\alpha 17$

Turn 8

$\alpha 18$

RNA NA NAN

LEVDRYLLNRLREFTASTINNYENFDYLNIYQEVQNFINVEL

Turn $9 \quad \alpha 19$

$\alpha 20$

R

SNFYLDYGKDLYIEQRDSHIRRSMQTVLYQILVDMTKLLAP 758 Loop 18

Turn10 $\quad 0.21$

ILVHTAEEVWSHTPHVKEESVHLADMPKVVEV

$\alpha 22$

$\beta 8$

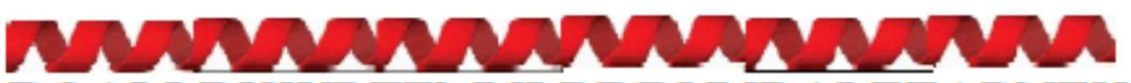

Loop 20 
$\beta 8$

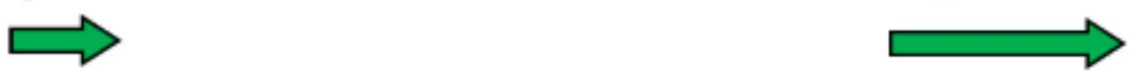

IASNDKFNASEFLTSFDALHQLFIVSQVKVVDKLDDQATAYEH 873

Loop 21

$\beta 10$

$\beta 11$ Turn $11 \beta 12$ Turn 12

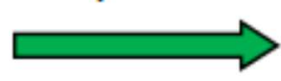

GDIVIEHADGEKCERCWNYSEDLGAVDELTHLC

Loop 23

$\alpha 23$

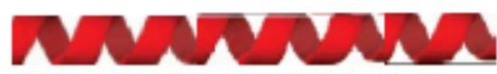

PRCQQVV KSLV

Fig. S2.The primary sequence includes loops (coloured in blue), helices (marked by red helical structure over the sequence, turns (marked by yellow lines), beta sheets (marked by green arrow). 
S3. Sequence alignment of ${ }^{S a}$ IleRS (1FFY_2 $\left.{ }^{24}, 1 \mathrm{QU} 3 \_2\right),{ }^{\mathrm{Ca}} \mathrm{IleRS}(6 \mathrm{LDK})^{25},{ }^{\text {Tt }}$ IleRS (1ILE_1, 1JZS) ${ }^{26}$,

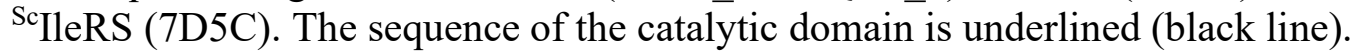

CLUSTAL O(1.2.4) multiple sequence alignment

\begin{tabular}{|c|c|}
\hline 1FFY_2 & Chain \\
\hline 1QU3_2 & Chain \\
\hline $\mathrm{sp} \mid \mathrm{B} \overline{\mathrm{T}} \mathrm{MO}$ & $\partial C 2.1 \mid$ SYI_ECO8A \\
\hline 1ILE_1 & Chain \\
\hline $1 J z Q 1$ & Chain \\
\hline 1JZS_1| & Chain \\
\hline 6LDK_1| & Chain \\
\hline 7D5C_1 & Chain \\
\hline 1FFY_2 & Chain \\
\hline 1QU3_2 & Chain \\
\hline sp|BतMO & $\partial C 2.1 \mid S Y I$ SCO8A \\
\hline 1ILE_1| & Chain \\
\hline $1 J Z Q 11$ & Chain \\
\hline 1JZS_11 & Chain \\
\hline 6LDK_1 & Chain \\
\hline 705C_1 & Chain \\
\hline 1FFY_2 & Chain \\
\hline 1QU3_2 & Chain \\
\hline sp|Bत̄MO & OC2.1|SYI_ECO8A \\
\hline 1ILE_1 & Chain \\
\hline $1 J z Q-1 \mid$ & Chain \\
\hline 1JZS_1| & Chain \\
\hline 6LDK_1| & Chain \\
\hline 7D5C_11 & Chair \\
\hline
\end{tabular}

-MDYEKTLLMPKTDFPMRGGLPNKEPQIQEKWDAEDQYHKALEKNKGNETFILHDGPPYA 59 -MDYEKTLLMPKTDFPMRGGLPNKEPQIQEKWDAEDQYHKALEKNKGNETFILHDGPPYA 59 MSDYKSTLNLPETGFPMRGDLAKREPGMLARWTDDDLYGIIRAAKKGKKTFILHDGPPYA 60 - - - -MFKEVGE - - - PNFPKLEEEVLAFWKREKIFQKSVENRKGGPRYTVYEGPPTA 49 - - - - MFKEVGE - PNFEEEVLAFWKREKIFQKSVENRKGGPRYTVYEGPPTA 49 - - - MFKEVGE-- - PNFPKLEEEVLAFWKREKIFQKSVENRKGGPRYTVYEGPPTA 49 MSLQESNNNIPQGA - - - -FSFPKEEEAVIKHWDDVNAFQRTLELTEDLPPFAFFDGPPFA 56 - - - -MSESNAH- - -FSFPKEEEKVLSLWDEIDAFHTSLELTKDKPEFSFFDGPPFA 49 .: : ${ }^{*}:$ * $^{*}$. : $\quad: \quad: \ldots: * * * *$

NGNLHMGHALNKILKDFIVRYKTMQGFYAPYVPGWDTHGLPIEQALTK-KGVDRK-- - -K 114 NGNLHMGHALNKILKDFIVRYKTMQGFYAPYVPGWDTHGLPIEQALTK-KGVDRK----K 114 NGSIHIGHSVNKILKDIIVKSKGLSGYDSPYVPGWDCHGLPIELKVEQEYGKPGE ----K 116 NGLPHVGHAQARSYKDLFPRYKTMRGYYAPRRAGWDTHGLPVELEVEKKLGLKSKREIEA 109 NGLPHVGHAQARSYKDLFPRYKTMRGYYAPRRAGWDTHGLPVELEVEKKLGLKSKREIEA 109 NGLPHVGHAQARSYKDLFPRYKTMRGYYAPRRAGWDTHGLPVELEVEKKLGLKSKREIEA 109 TGTPHYGHILASTVKDIIPRYATMNGYHVERRFGWDTHGLPVEHEIDKKLNITSKEDVYA 116 TGTPHYGHILASTIKDIVPRYATMTGHHVERRFGWDTHGVPIEHIIDKKLGITGKDDVFK 109 $.^{*} * * * \quad * *: .::^{*} . \quad * * * * *:^{*}: *:$ :

MSTAEFREKCKEFALEQIELQKKDFRRLGVRGDFNDPYITLKPEYEAAQIRIFGEMADKG 174 MSTAEFREKCKEFALEQIELQKKDFRRLGVRGDFNDPYITLKPEYEAAQIRIFGEMADKG 174 FTAAEFRAKCREYAATQVDGQRKDFIRLGVLGDWSHPYLTMDFKTEANIIRALGKIIGNG 176 YGIERFNQACRESVFTYEKEWEAFTERIAYWVDLEDAYATLEPTYIESIWWSLKNLFDRG 169 YGIERFNQACRESVFTYEKEWEAFTERIAYWVDLEDAYATLEPTYIESIWWSLKNLFDRG 169 YGIERFNQACRESVFTYEKEWEAFTERIAYWVDLEDAYATLEPTYIESIWWSLKNLFDRG 169 MGIDKYNAECRAIVMRYADEWRRTIKRLGRWIDMDNDYKTLYPEFMESWWWAFKELFNKD 176 YGLENYNNECRSIVMTYASDWRKTIGRLGRWIDFDNDYKTMYPSFMESTWWAFKQLHEKG 169
.:. * :
$::: \quad$. 
1FFY_2|Chain

1QU3_2|Chain

sp|B7MEC2.1|SYI_ECO8A

1ILE_1|Chain

1JZQ 1|Chain

1JZS 1|Chain

6LDK_1|Chain

7D5C_1|Chain

1FFY_2|Chain 1QU3_2|Chain Sp|B7MOC2.1|SYI_ECO8A

1ILE_1|Chain

1JZ0 1 Chain

1JZS_1|Chain

6LDK_1|Chain

7D5C_1|Chain

1FFY_2|Chain

1QU3_2|Chain

Sp|B7MOC2.1|SYI_ECO8A

1ILE_1|Chain

1JZQ 1 Chain

1JZS 1|Chain

6LDK_1|Chain

7DSC_1|Chain
LIYKGKKPVYWSPSSESSLA--EAEIEYHDKRSASIYVAFNVKDDKG---.---VVDAD 224

LIYKGKKPVYWSPSSESSLA--EAEIEYHDKRSASIYVAFNVKDDKG--.----VVDAD 224 HLHKGAKPVHWCVDCRSALA--EAEVEYYDKTSPSIDVAFQAVDQDALKAKFAVSNVNGP 234 LLYRDHKVVPYCPRCGTPLSSHEVALGYKEIQDPSWYVRFPLKEPKK--.---_LGEK 221 LLYRDHKVVPYCPRCGTPLSSHEVALGYKEIQDPSVYVRFPLKEPKK--.--LGLEK 221 LLYRDHKVVPYCPRCGTPLSSHEVALGYKEIQDPSWYVRFPLKEPKK--..-- LGLEK 221 AVYRGLRVMPYSTACTTPLSNFEAQQNYKEVNDPALTISFPLL

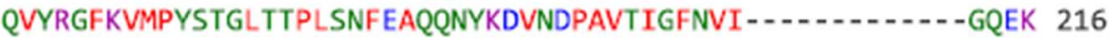

$$
::: \text { : : : . : * * * * : . : : : * }
$$

AKFIIWTTTPWTIPSNVAITVHPELKYGQYNVN--GEKYIIAEALSDAVAEALDWDKASI 282 AKFIIWTTTPWTIPSNVAITVHPELKYGQYNVN--GEKYIIAEALSDAVAEALDWDKASI 282 ISLVIWTTTPWTLPANRAISIAPDFDYALVQID--GQAVILAKDLVESVMQRIGVTD--Y 290 ASLLIWTTTPWTLPGNVAAAVHPEYTYAAFQVG--DEALILEEGLGR----KLLG--EGT 273 ASLLIWTTTPWTLPGNVAAAVHPEYTYAAFQVG--DEALILEEGLGR----KLLG--EGT 273 ASLLIWTTTPWTLPGNVAAAVHPEYTYAAFQVG--DEALILEEGLGR----KLLG--EGT 273 TCLVAWTTTPWTLPANLALAVNPKFEYVKIFDEEKKKNFILLESLISTLYKKPKS--AKF 281 TQLVAWTTTPWTLPSNLSLCVNADFEYVKIYDETRDRYFILLESLIKTLYKKPKN--EKY 274 : : * : : *

KLEKEYTGKELEWVVAQHPF - - - - - LDRESLVINGDHVTTDAGTGCVHTAPGHGEDDY 335 KLEKEYTGKELEWWVAQHPF--- - - LDRESLVINGDHVTTDAGTGCVHTAPGHGEDDY 335 TILGTVKGAELELLRFTHPF - - - - - - MGFDVPAILGDHVTLDAGTGAVHTAPGHGPDDY 343 QVLKTFPGKALEGLPYTPPYPQ----ALEKGYFVVLADYVSQEDGTGIVHQAPAFGAEDL 329 QVLKTFPGKALEGLPYTPPYPQ----ALEKGYFVLLADYVSQEDGTGIVHQAPAFGAEDL 329 QVLKTFPGKALEGLPYTPPYPQ----ALEKGYFWLADYVSQEDGTGIVHQAPAFGAEDL 329 KVVEKILGKDLVGLKYKPLFNYFYEDFKDTGFRVIPADYVTNDSGTGIVHQAPSYGEEDF 341 KIVEKIKGSDLVGLKYEPLFPYFAEQFHETAFRVISDDYVTSDSGTGIVHNAPAFGEEDN 334

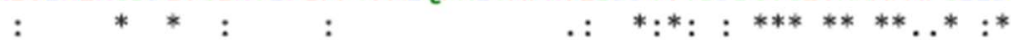




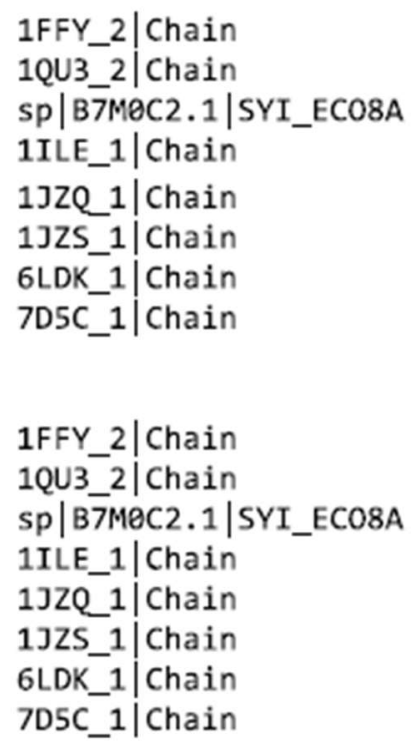

1FFY_2|Chain 1QU3_2|Chain Sp|B7MOC2.1|SYI_ECO8A

1ILE_1|Chain

1JZQ 1|Chain

1JZS_1|Chain

6LDK 1 Chain

7D5C_1|Chain

1FFY_2|Chain 1QU3_2|Chain sp|B7M8C2.1|SYI_ECO8A

1ILE_1|Chain

1JZQ 1|Chain

1JZS_1|Chain

6LDK_1|Chain

7D5C_1|Chain
IVGQQYELPVI-- - -SPIDDKGVFTEE-GGQFEGMFYDKANKAVTDLLTEKGALLKLDF 389 IVGQQYELPVI-----SPIDDKGVFTEE-GGQFEGMFYDKANKAVTDLLTEKGALLKLDF 389 VIGQKYGLETA-----NPVGPDGTYLPGTYPTLDGVNVFKANDIWVALLQEKGALLHVEK 398 ETARVYGLPLL----KTVDEEGKL--L-VEPFKGLYFREANRAILRDLRGRGLLFKEES 381 ETARVYGLPLL - - --KTVDEEGKL - - L-VEPFKGLYFREANRAILRDLRGRGLLFKEES 381 ETARVYGLPLL -----KTVDEEGKL--L-VEPFKGLYFREANRAILRDLRGRGLLFKEES 381 NSTKAAGVINEKKLPPSIVDDSGRMESN-VPEIAGMYFKDADKVIIKKLSEEGRLLVNTQ 400 AACLKNGVISEDSVLPNAIDDLGRFTKD-VPDFEGVYVKDADKLIIKYLTNTGNLLLASQ 393

ITHSYPHDWRTKKPVIFRATPQWFASISK - -VRQDILDAIENTNFKVNWG-KTRIYNMVR 446 ITHSYPHDWRTKKPVIFRATPQWFASISK--VRQDILDAIENTNFKVNWG-KTRIYNMVR 446 MQHSYPCCWRHKTPIIFRATPQWFVSMDQKGLRAQSLKEIKGVQWIPDWG-QARIESMVA 457 YLHSYPHCWRCSTPLMYYATESWFIKNTL - -FKDELIRNNQE IHWVPPHIKEGRYGEWLK 439 YLHSYPHCWRCSTPLMYYATESWFIKNTL - -FKDELIRNNQE IHWVPPHIKEGRYGEWLK 439 YLHSYPHCWRCSTPLMYYATESWFIKNTL - -FKDELIRNNQE IHWVPPHIKEGRYGEWLK 439 VKHSYPFCWRSDTPLMYRTVPAWFVRIGE - -VIPEMLDNVEKTNWVPSNIKDKRFSNWIA 458 IRHSYPFCWRSDTPLLYRSVPAWFVRVKN - -IVPQMLDSVMKSHWVPNTIKEKRFANWIA 451

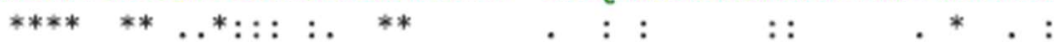

DRGEWVISRORVWGVPLPVFYAENGEIIMTK - -ETVNHVADLFAEHGS - -NIWFEREAKD 502 DRGEWVISRQRVWGVPLPVFYAENGEIIMTK--ETVNHVADLFAEHGS--NIWFEREAKD 502 NRPDWCISRQRTWGVPMSLFVHKDTEELHPRTLELMEEVAKRVEVDGI--QAWWDLDAKE 515 NLVDWALSRNRYWGTPLPIWVCQACGKEEAI-- - -GSFQELKARATKPLPEPFDPHR -P 493 NLVDWALSRNRYWGTPLPIWIVCQACGKEEAI-----GSFQELKARATKPLPEPFDPHR -P 493 NLVDWALSRNRYWGTPLPIWVCQACGKEEAI-----GSFQELKARATKPLPEPFDPHR-P 493 NARDWNISRNRYWGTPIPLWVSDDFEEMVCV-----GSIQELRELSG--RDDITDIHR-E 510 NARDWINVSRNRYWGTPIPLWVSDDFEEVVCV-----GSIKELEELTG--VRNITDLHR-D 503 : $:^{*}:^{* *}:^{* * *} .^{*}:$ : : . . .

LLPEGFTHP-GSPNGTFTKETDIMDVWFDSGSSHRGV- - - - LETRPELS-FPADMYLEG 555 LLPEGFTHP-GSPNGTFTKETDIMDVWFDSGSSHRGV- - - -LETRPELS-FPADMYLEG 555 ILG--.--DEADQYVKVPDTLDVWFDSGSTHSSV--.--VDVRPEFAGHAADMYLEG 562 YV-DQVELACA-CGGTMRRVPYVIDVWYDSGAMPFASLHYPFEHEEVFRESFPADFIAEG 551 YV-DQVELACA-CGGTMRRVPYVIDVWYDSGAMPFASLHYPFEHEEVFRESFPADFIAEG 551 YV-DQVELACA-CGGTMRRVPYVIDVWYDSGAMPFASLHYPFEHEEVFRESFPADFIAEG 551 SI-DSITIPSKKGKGQLKRIEEVFDCWFESGSMPYASKHYPFENEKKFLDAFPANFISEG 569 VI-DKLTIPSKQGKGDLKRIEEVFDCWFESGSMPYASQHYPFENTEKFDERVPANFISEG 562

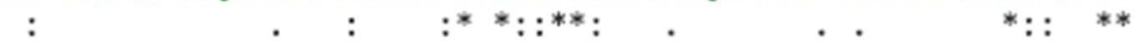


1FFY_2|Chain 1QU3_2|Chain sp|B7MEC2.1|SYI_ECO8A 1ILE_1|Chain 1JZQ 1 Chain 1JZS_1|Chain 6LDK 1 Chain 7D5C_1|Chain

1FFY_2
1QU3_2 Chain
SP|B7MOC2.1 $\mid$ SYI_ECO8A
1ILE_1 $\mid$ Chain
1JZQ11 Chain
1JZS_1 Chain
6LDK_1 Chain
7DSC_1 Chain

1FFY_2 Chain 1QU3_2|Chain Sp|B7MOC2.1|SYI_ECO8A

1ILE_1|Chain

1JZQ 1 Chain

1JZS 1 Chain 6LDK_1|Chain 7D5C_1|Chain

SDQYRGWFNSSITTSVATRGVSPYKFLLSHGFVMDGEGKKMSKSLGNVIVPDQVVKQKGA 615 SDQYRGWFNSSITTSVATRGVSPYKFLLSHGFVMDGEGKKMSKSLGNVIVPDQVVKQKGA 615 SDQHRGWFMSSLMISTAMKGKAPYRQVLTHGFTVDGQGRKMSKSIGNTVSPQDVMNKLGA 622 IDQTRGWFNSLHQLGVMLFGSIAFKNVICHGLILDEKGQKMSKSKGNVVDPWDIIRKFGA 611 IDQTRGWFNSLHQLGVMLFGSIAFKNVICHGLILDEKGQKMSKSKGNVVDPWDIIRKFGA 611 IDQTRGWFNSLHQLGVMLFGSIAFKNVICHGLILDEKGQKMSKSKGNVVDPWDIIRKFGA 611 LDQTRGWFYTLTVLGTHLFKTAPYONVIVTGIVLAADGKKMSKRLKNYPDPTLVLEKYGA 629 LDQTRGWFYTLAVLGTHLFGSVPYKNVIVSGIVLAADGRKMSKSLKNYPDPSIVLNKYGA 622

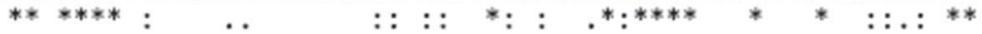

DIARLWVSSTDYLA--DVRISDEILKQTSD-DYRKIRNTLRFMLGNINDFNPD--T----D 667 DIARLWVSSTDYLA--DVRISDEILKQTSD-DYRKIRNTLRFMLGNINDFNPD--T---D 667 DILRLWVASTDYTG--EMAVSDEILKRAAD-SYRRIRNTARFLLANLNGFDPA--K---D 674 DALRWYIYVSAPPEA-DRRFGPNLVRETVRDYFLTLWNVYSFFVTYANLDR-- - PDLKN 666 DALRWYIYVSAPPEA-DRRFGPNLVRETVRDYFLTLWNVYSFFVTYANLDR----PDLKN 666 DALRWYIYVSAPPEA-DRRFGPNLVRETVRDYFLTLWNVYSFFVTYANLDR----PDLKN 666 DALRLYLINSPVLRAETLKFKEEGVKEIVSSVLLPWYNSYKFLKDAADLFKKDNGKDFVY 689 DALRLYLINSPVLKAESLKFKEEGVKEWSKVLLPWWNSFKFLDGQIALLKKMSNIDFQY 682 * * : : : $:$ : : : : * * :

SIPESELLEVDRYLLNRLREFTASTINNYENFDYLNIYQEVQNFINVELSNFYLDYGKDI 727 SIPESELLEVDRYLLNRLREFTASTINNYENFDYLNIYQEVQNFINVELSNFYLDYGKDI 727 MVKPEEMVVLDRWAVGCAKAAQEDILKAYEAYDFHEVVQRLMRFCSVEMGSFYLDIIKDR 734 PPPPEKRPEMDRWLLARMQDLIQRVTEALEAYDPTTSARALRDFVVEDLSQWYVRRNRRR 726 PPPPEKRPEMDRILLLARMQDLIQRVTEALEAYDPTTSARALRDFWVDLSQWYVRRNRRR 726 PPPPEKRPEMDRWLLARMQDLIQRVTEALEAYDPTTSARALRDFVVDLSQWYVRRNRRR 726 DNSLHSTNVMDRWLLASIQSLIKFIHEEMTGYRLYTVVPRLLHF-IDDLTNWYIRFNRRR 748 DDSVKSDNVMDRWILASMQSLVQFIHEEMGQYKLYTVVPKLLNF-IDELTNWYIRFNRRR 741
$:^{* *}::$ : 


\author{
1FFY_2|Chain \\ 1QU3_2|Chain \\ Sp $|B \overline{7} M O C 2.1|$ SYI_ECO8A \\ 1ILE_1|Chain \\ 1JZQ 1|Chain \\ 1JZS_1|Chain \\ 6LDK_1|Chain \\ 7D5C_1|Chain
}

1FFY_2|Chain
1QU3_2|Chain
Sp|B7MOC2.1|SYI_ECO8A
1ILE_1|Chain
1JZQ_1|Chain
1JZS_1|Chain
6LDK_1|Chain
7D5C_1|Chain

1FFY_2|Chain 1QU3_2|Chain SP|BTMOC2.1|SYI_ECO8A 1ILE_1|Chain 1JZQ_1|Chain 1JZS_1|Chain 6LDK_1|Chain 7D5C_1|Chain

1FFY_2 Chain
1QU3_2 $\mid$ Chain
Sp $\mid$ B7MBC2.1 SYIECO8A
1ILE_1 Chain
1JZQ_1 Chain
1JZS_1 Chain
6LDK_1 Chain
7D5C_1 Chain

LYIEQR-DSHIRRSMQTVLYQILVDMTKLLAPILVHTAEEVWSHTPLYIEQR-DSHIRRSMQTVLYQILVDMTKLLAPILVHTAEEVWSHTP _. QYTAKA-DSVARRSCQTALYHIAEALVRWMAPILSFTADEVWGYLP FWKN - EDALDREAAYATLYEALVLVATLAAPFTPFLAEVLIVQNLVRSVRL - _ - _ - 775 FWKN - -EDALDREAAYATLYEALVLVATLAAPFTPFLAEVLWQNLVRSVRL - _ - - - - - - 775 FWKN - -EDALDREAAYATLYEALVLVATLAAPFTPFLAEVLWQNLVRSVRL - _. - - - 775 IKGYASEDVEDTQKGLNTLVEALLTLSRAMAPFTPYLADGIYQRIKVYFKQEDLEKIAIN 808 LKGE - -NGVEDCLKALNSLFDALFTFVRAMAPFTPFLSESIYLRLKEYIPEAVLAKY- - - 796 **: . : : : :

- -HVKEESVHLADMP-- - -KVVEVDQALLDKWRTFMNLRDDVNRALETARNEKVI-GKSL 825 --HVKEESVHLADMP----KVVEVDQALLDKWRTFMNLRDDVNRALETARNEKVI-GKSL 825 --GEREKYVFTGEWYEGLFGLADSEAMNDAFWDELLKVRGEVNKVIEQARADKKV-GGSL 836 - - - EAKESVHLADWPEADPALADEA - - L - - - - - -VAQMRAVLKVVDLARAARAKSGV - 821 - - -EAKESVHLADWPEADPALADEA - - L - - - - -VAQMRAVLKVVDLARAARAKSGV - 821 - - -EAKESVHLADWPEADPALADEA - - L - - - - -VAQMRAVLKVVDLARAARAKSGV- 821 PKNVDLRSVHFLSYPSVRQELFDEK-- I - - - - - -EVAVARMQKVIDMARNIREKKM- - - 856 --GKDGRSVHFLSYPVVKKEYFDEA-- I - - - - - -ETAVSRMQSVIDLGRNIREKKTISL 845
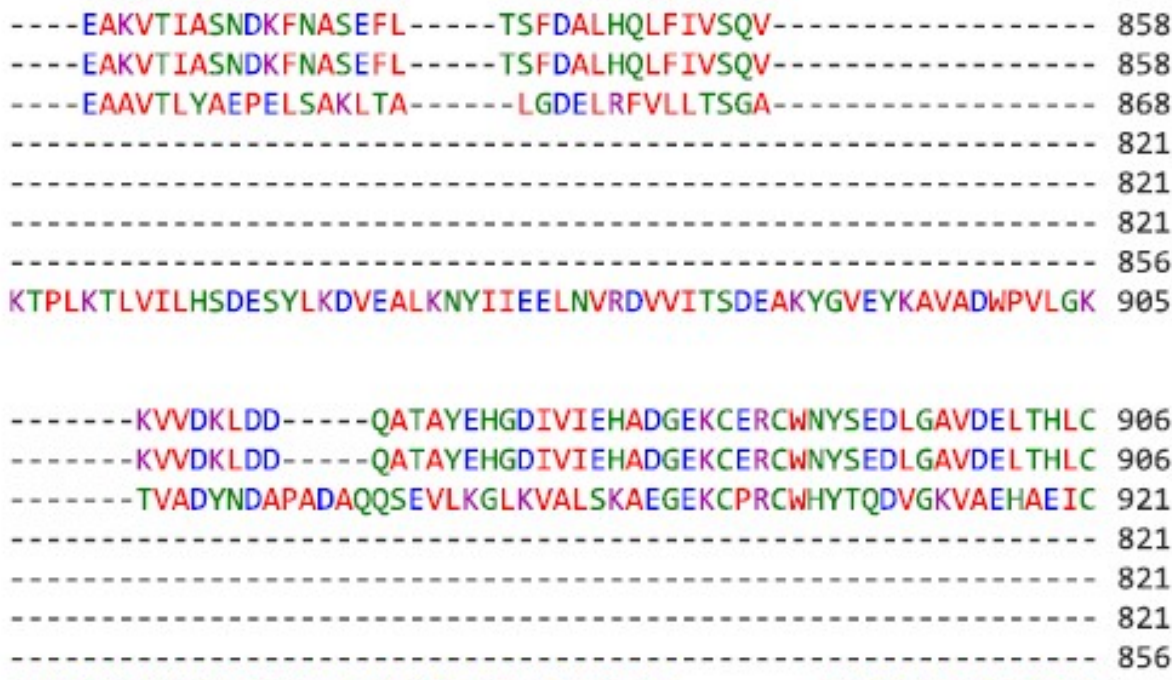

KLKKDAKKVKDALPSVTSEQVREYLESGKLEVAGIEL -...-. VKGDLNAIRGLPESA 957

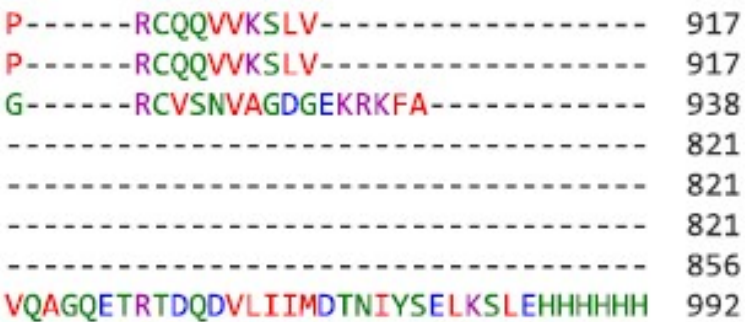

Fig. S3. Sequence alignment of ${ }^{S a} \mathrm{IleRS}\left(1 \mathrm{FFY} \_2^{24}, 1 \mathrm{QU} 3 \_2\right),{ }^{C a} \mathrm{IleRS}(6 \mathrm{LDK})^{25},{ }^{T t} \mathrm{IleRS}\left(1 \mathrm{ILE} \_1,1 \mathrm{JZS}\right)^{26}$, ${ }^{\mathrm{Sc}}$ IleRS (7D5C). The sequence of the catalytic domain is underlined (black line). 
S4. Summary of simulations carried out in the present work

Table S4. (a)

\begin{tabular}{|c|c|c|c|c|}
\hline \multicolumn{5}{|c|}{ No. of atoms in the simulated states of Sa IleRS studied in the present work } \\
\hline & $\begin{array}{l}\text { No. of } \\
\text { heavy } \\
\text { atoms in } \\
\text { aaRS }\end{array}$ & $\begin{array}{l}\text { No. of } \\
\text { heavy } \\
\text { atoms in } \\
\text { tRNA }\end{array}$ & $\begin{array}{l}\text { No. of } \\
\text { water }\end{array}$ & $\begin{array}{l}\text { Total no. of atoms } \\
\text { including, ions }\left(\mathrm{Mg}^{+2},\right. \\
\left.\mathrm{Cl}^{-}, \mathrm{Na}^{+}, \mathrm{Zn}^{+2}\right) \text { and } \\
\text { hydrogens }\end{array}$ \\
\hline${ }^{\text {SaIleRS (open) }}$ & 7407 & - & 75758 & 241964 \\
\hline $\begin{array}{l}\text { SaIleRS:tRNA:Ile- } \\
\text { AMP }\end{array}$ & 7407 & 1644 & 74431 & 240635 \\
\hline SaIleRS:tRNA:MRC & 7407 & 1644 & 65020 & 212425 \\
\hline $\begin{array}{l}\text { SaIleRS(V588F): } \\
\text { tRNA:MRC }\end{array}$ & 7407 & 1644 & 65023 & 212435 \\
\hline $\begin{array}{l}\text { SaIleRS tRNA:MRC } \\
\text { Metadynamics }\end{array}$ & 7407 & 1644 & 65020 & 212425 \\
\hline
\end{tabular}

Table S4. (b)

\begin{tabular}{|l|l|l|l|c|}
\hline \multicolumn{2}{|c|}{$\begin{array}{l}\text { Simulated states of SaleRS studied in the present } \\
\text { work }\end{array}$} & \multicolumn{2}{c|}{ Duration of simulation (ns) } \\
\hline $\begin{array}{l}\text { aaRS } \\
\text { (state of the active site) }\end{array}$ & tRNA & Substrate & $\begin{array}{l}\text { Duration } \\
\text { of the } \\
\text { production } \\
\text { run }\end{array}$ & $\begin{array}{l}\text { Duration of trajectory } \\
\text { length used for the } \\
\text { analysis presented in } \\
\text { this work }\end{array}$ \\
\hline $\begin{array}{l}\text { SaIleRS } \\
\text { (Open state) }\end{array}$ & Absent & Absent & 30 & 20 \\
\hline $\begin{array}{l}\text { SaleRS } \\
\text { (Closed state) }\end{array}$ & tRNA & Ile-AMP & 30 & 20 \\
\hline $\begin{array}{l}\text { SaIleRS } \\
\text { (Closed state) }\end{array}$ & tRNA & Mupirocin & 30 & 20 \\
\hline $\begin{array}{l}\text { SaIleRS(V588F) } \\
\text { (Closed state; mutated) }\end{array}$ & tRNA & Mupirocin & 20 & \\
\hline $\begin{array}{l}\text { SaIleRS } \\
\text { Metadynamics } \\
\text { (Unbinding of MRC } \\
\text { from the closed state) }\end{array}$ & tRNA & Mupirocin & 80 & \\
\hline
\end{tabular}


S5.

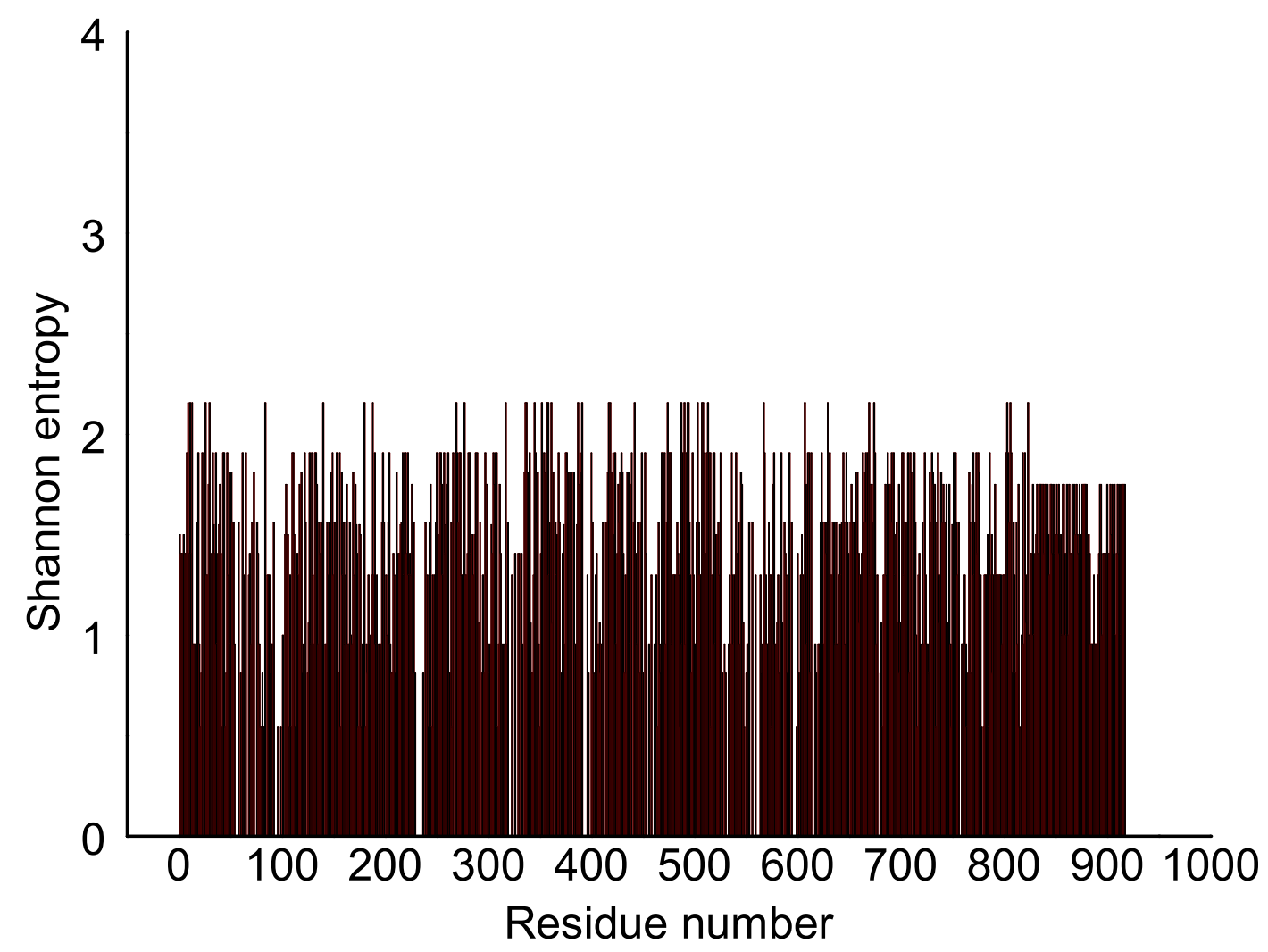

Fig. S5. Plot of Shannon entropy of the sequence alignment of ${ }^{S a}$ IleRS (1FFY_2) ${ }^{24},{ }^{C a}$ IleRS (6LDK) ${ }^{25}$, ${ }^{T}$ IleRS $(1 \mathrm{JZS})^{26}$ against the residue number. 
S6.

Table S6. The conserved residues in sequences of IleRS from different organisms mentioned in the text.

\begin{tabular}{|c|c|c|c|c|c|c|c|}
\hline $1 F F Y . p d b$ & $1 Q U 3 . p d b$ & $S Y I \_E C O 8 A$ & IILE.pdb & 1JZQ.pdb & 1JZS.pdb & 6LDK.pdb & 7D5C.pdb \\
\hline Glu24 & Glu24 & Glu25 & Glu14 & Glu14 & Glu14 & Glu21 & Glu14 \\
\hline Trp31 & Trp31 & Trp32 & Trp21 & Trp21 & Trp21 & Trp28 & Trp21 \\
\hline Gly55 & Gly55 & Gly56 & Gly45 & Gly45 & Gly45 & Gly52 & Gly45 \\
\hline Pro56 & Pro56 & Pro57 & Pro46 & Pro46 & Pro46 & Pro53 & Pro46 \\
\hline Pro57 & Pro57 & Pro58 & Pro47 & Pro47 & Pro47 & Pro54 & Pro47 \\
\hline Ala59 & Ala59 & Ala60 & Ala49 & Ala49 & Ala49 & Ala56 & Ala49 \\
\hline Gly61 & Gly61 & Gly62 & Gly51 & Gly51 & Gly51 & Gly58 & Gly51 \\
\hline His64 & His64 & His65 & His54 & His54 & His54 & His61 & His54 \\
\hline Gly66 & Gly66 & Gly67 & Gly56 & Gly56 & Gly56 & Gly63 & Gly56 \\
\hline His67 & His67 & His68 & His57 & His57 & His57 & His64 & His57 \\
\hline Lys74 & Lys74 & Lys75 & Lys64 & Lys64 & Lys64 & Lys71 & Lys64 \\
\hline Asp75 & Asp75 & Asp76 & Asp65 & Asp65 & Asp65 & Asp72 & Asp65 \\
\hline Gly 85 & Gly85 & Gly86 & Gly75 & Gly75 & Gly75 & Gly82 & Gly75 \\
\hline Gly93 & Gly93 & Gly94 & Gly83 & Gly83 & Gly83 & Gly90 & Gly83 \\
\hline Trp94 & Trp94 & Trp95 & Trp84 & Trp84 & Trp84 & Trp91 & $\operatorname{Trp} 84$ \\
\hline Asp95 & Asp95 & Asp96 & Asp85 & Asp85 & Asp85 & Asp92 & Asp85 \\
\hline His97 & His97 & His98 & His87 & His87 & His87 & His94 & His87 \\
\hline Gly98 & Gly98 & Gly99 & Gly88 & Gly88 & Gly88 & Gly96 & Gly88 \\
\hline Pro100 & Pro100 & Pro101 & Pro90 & Pro90 & Pro90 & Pro97 & Pro90 \\
\hline Glu102 & Glu102 & Glu103 & Glu92 & Glu92 & Glu92 & Glu99 & Glu92 \\
\hline Cys124 & Cys124 & Cys 126 & Cys119 & Cys119 & Cys119 & Cys126 & Cys119 \\
\hline Arg 141 & $\operatorname{Arg} 141$ & Arg 143 & $\operatorname{Arg} 136$ & $\operatorname{Arg} 136$ & $\operatorname{Arg} 136$ & Arg 143 & $\operatorname{Arg} 136$ \\
\hline Asp147 & Asp147 & Asp149 & Asp142 & Asp142 & Asp142 & Asp149 & Asp142 \\
\hline Tyr152 & Tyr152 & Tyr154 & Tyr147 & Tyr147 & Tyr147 & Tyr154 & Tyr147 \\
\hline Thr154 & Thr154 & Thr156 & Thr149 & Thr149 & Thr149 & Thr156 & Thr149 \\
\hline Trp412 & Trp412 & Trp421 & Trp404 & Trp404 & Trp404 & Trp423 & Trp416 \\
\hline Phe413 & Phe413 & Phe422 & Phe405 & Phe405 & Phe405 & Phe424 & Phe417 \\
\hline Arg440 & $\operatorname{Arg} 440$ & $\operatorname{Arg} 451$ & $\operatorname{Arg} 433$ & $\operatorname{Arg} 433$ & $\operatorname{Arg} 433$ & Arg452 & Arg445 \\
\hline Trp451 & Trp451 & Trp462 & Trp444 & Trp444 & Trp444 & Trp463 & Trp456 \\
\hline Ser454 & Ser454 & Ser465 & Ser447 & Ser447 & Ser447 & Ser466 & Ser459 \\
\hline $\operatorname{Arg} 455$ & $\operatorname{Arg} 455$ & $\operatorname{Arg} 466$ & $\operatorname{Arg} 448$ & $\operatorname{Arg} 448$ & $\operatorname{Arg} 448$ & $\operatorname{Arg} 467$ & $\operatorname{Arg} 460$ \\
\hline Arg457 & Arg457 & Arg468 & Arg450 & Arg450 & Arg450 & Arg469 & $\operatorname{Arg} 462$ \\
\hline \begin{tabular}{|l|} 
Trp459 \\
\end{tabular} & Trp459 & Trp470 & Trp452 & Trp452 & Trp452 & Trp471 & Trp464 \\
\hline Gly460 & Gly460 & Gly471 & Gly453 & Gly453 & Gly453 & Gly472 & Gly465 \\
\hline Pro462 & Pro462 & Pro473 & Pro455 & Pro455 & Pro455 & Pro474 & Pro467 \\
\hline Trp528 & Trp528 & Trp534 & Trp518 & Trp518 & Trp518 & Trp536 & Trp529 \\
\hline Ser531 & Ser531 & Ser537 & Ser521 & Ser521 & Ser521 & Ser539 & Ser532 \\
\hline Gly532 & Gly532 & Gly538 & Gly522 & Gly522 & Gly522 & Gly540 & Gly533 \\
\hline Ala549 & Ala549 & Ala556 & Ala545 & Ala545 & Ala545 & Ala563 & Ala556 \\
\hline Glu554 & Glu554 & Glu561 & Glu550 & Glu550 & Glu550 & Glu568 & Glu561 \\
\hline \begin{tabular}{|l} 
Gly555 \\
\end{tabular} & Gly555 & Gly562 & Gly551 & Gly551 & Gly551 & Gly569 & Gly562 \\
\hline Asp557 & Asp557 & Asp564 & Asp553 & Asp553 & Asp553 & Asp571 & Asp564 \\
\hline Gln558 & Gln558 & Gln565 & Gln554 & Gln554 & Gln554 & Gln572 & Gln565 \\
\hline
\end{tabular}




\begin{tabular}{|l|l|l|l|l|l|l|l|}
\hline Arg560 & Arg560 & Arg567 & Arg556 & Arg556 & Arg556 & Arg574 & Arg567 \\
\hline Gly561 & Gly561 & Gly568 & Gly557 & Gly557 & Gly557 & Gly575 & Gly568 \\
\hline Trp562 & Trp562 & Trp569 & Trp558 & Trp558 & Trp558 & Trp576 & Trp569 \\
\hline Phe563 & Phe563 & Phe570 & Phe559 & Phe559 & Phe559 & Phe577 & Phe570 \\
\hline Gly586 & Gly586 & Gly593 & Gly582 & Gly582 & Gly582 & Gly600 & Gly593 \\
\hline Gly593 & Gly593 & Gly600 & Gly589 & Gly589 & Gly589 & Gly607 & Gly600 \\
\hline Lys595 & Lys595 & Lys602 & Lys591 & Ly5591 & Lys591 & Lys609 & Lys602 \\
\hline Met596 & Met596 & Met603 & Met592 & Met592 & Met592 & Met610 & Met603 \\
\hline Ser597 & Ser597 & Ser604 & Ser593 & Ser593 & Ser593 & Ser611 & Ser604 \\
\hline Lys598 & Lys598 & Lys605 & Lys594 & Lys594 & Lys594 & Lys612 & Lys605 \\
\hline Asn602 & Asn602 & Asn609 & Asn598 & Asn598 & Asn598 & Asn616 & Asn609 \\
\hline
\end{tabular}


S7.

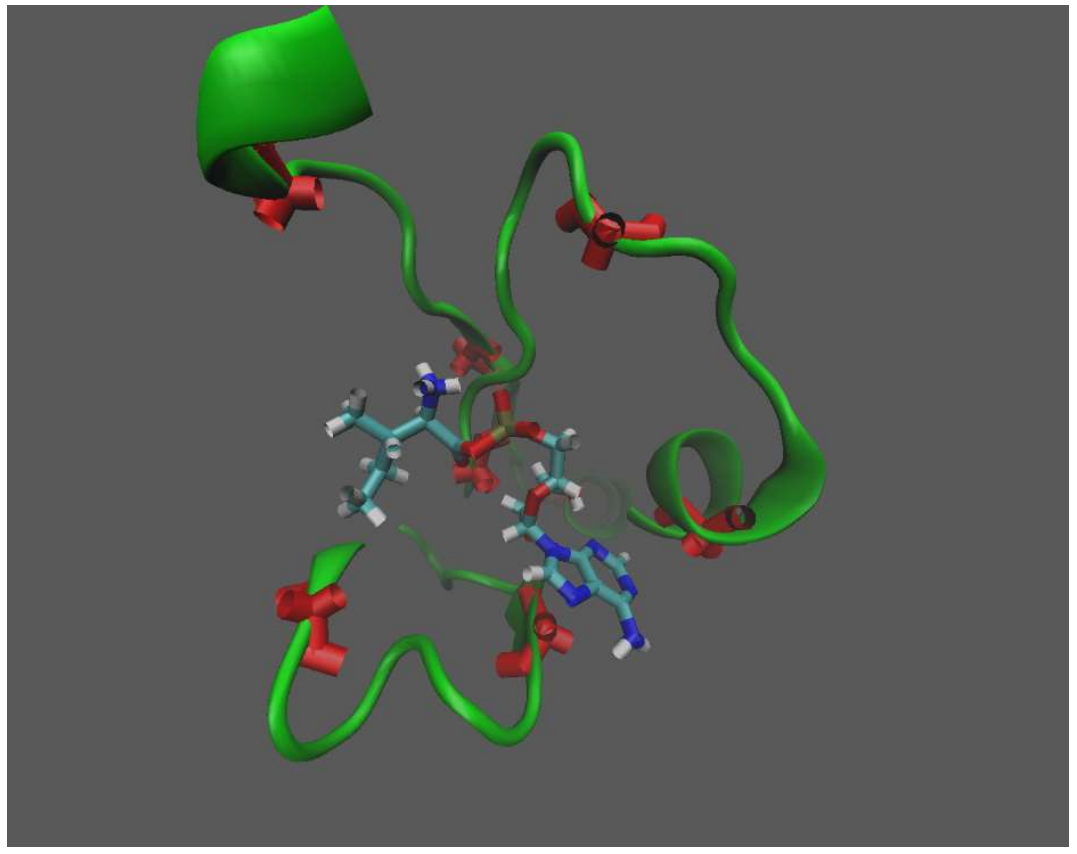

(a)

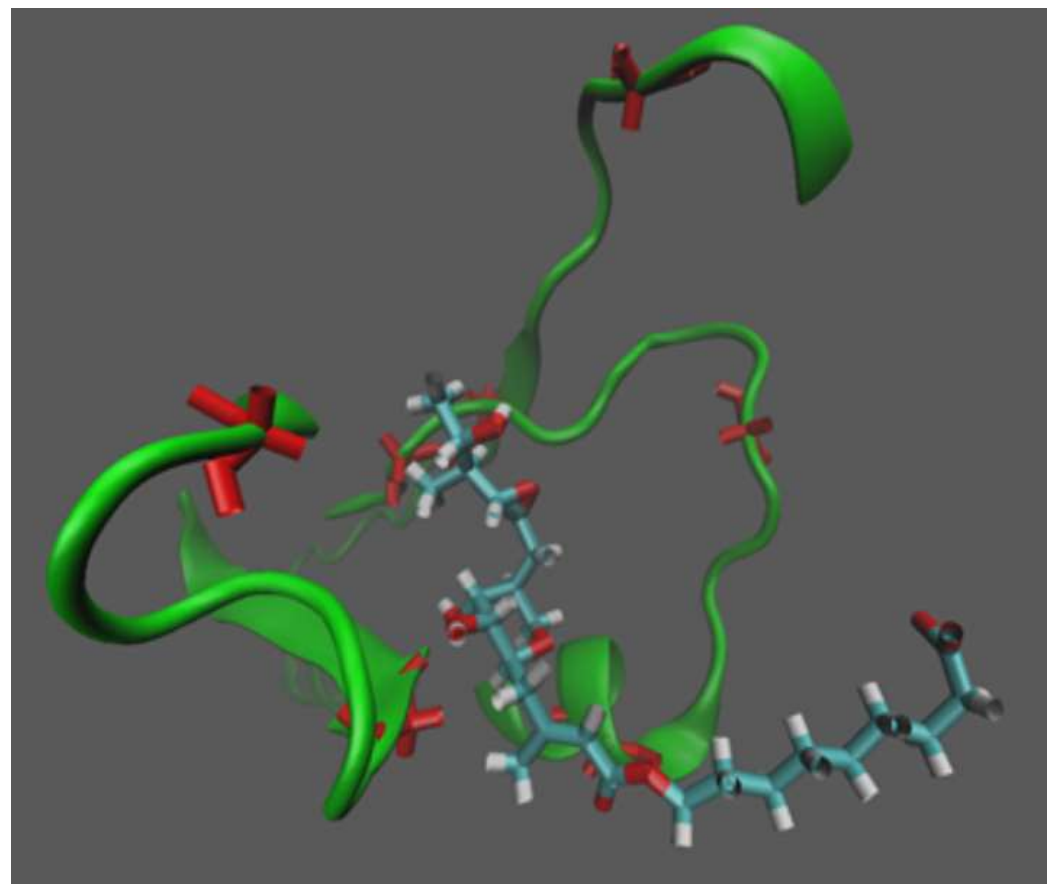

(b)

Fig. S7. The Gly residues (coloured in red) in HMGH and KMSKS loops in (a) Ile-AMP and (b) MRC bound system. 
1FFY_2|Chain 6LDK_1|Chain 1JZQ_1|Chain

1FFY_2|Chain 6LDK_1|Chain 1JZQ 1|Chain

1FFY_2|Chain 6LDK_1|Chain 1JZQ_1|Chain

1FFY_2|Chain 6LDK_1|Chain 1JZQ_1|Chain
MDYEK -TLLMPKTDFPMRGGLPNKEPQIQEKWDAEDQYHKALEKNKGNETFILHDGPPYA MSLQESNNNIPQ - - -GAFSFPKEEEAVIKHWDDVNAFQRTLELTEDLPPFAFFDGPPFA - - - - - -MFKEV - - - GEPNFPKLEEEVLAFWKREKIFQKSVENRKGGPRYTVYEGPPTA $. *^{*}: *^{*} * \quad .: \therefore:: * \quad: . \quad$ : .:****

NGNLHMGHALNKILKDFIVRYKTMQGFYAPYVPGWDTHGLPIEQALTKKGV- - - -DRKK TGTPHYGHILASTVKDIIPRYATMNGYHVERRFGWDTHGLPVEHEIDKKLNITSKEDVYA NGLPHVGHAQARSYKDLFPRYKTMRGYYAPRRAGWDTHGLPVELEVEKKLGLKSKREIEA .* * ** **: : ****.*: : $* * * * * * * *$ :

MSTAEFREKCKEFALEQIELQKKDFRRLGVRGDFNDPYITLKPEYEAAQIRIFGEMADKG MGIDKYNAECRAIVMRYADEWRRTIKRLGRWIDMDNDYKTLYPEFMESVWWAFKELFNKD YGIERFNQACRESVFTYEKEWEAFTERIAYWVDLEDAYATLEPTYIESIWWSLKNLFDRG . . . * * : . . .* : * : : * *** : : : : : :

LIYKGKKPVYWSPSSESSLA- -EAEIEYHDKRSASIYVAFNVKDDKGVVDADAKFIIWTT AVYRGLRVMPYSTACTTPLSNFEAQQNYKEVNDPALTISFPLLDN-- - - EDTCLVAWTT LLYRDHKVVPYCPRCGTPLSSHEVALGYKEIQDPSVYVRFPLKEPKKLGLEKASLLIWTT :*: : : : . : * * * *: . : : : * : : .: : :***
59

TPWTIPSNVAITVHPELKYGQYNVNGE - -KYIIAEALSDAVAEALDWDKASIKLEKEYTG TPWTLPANLALAVNPKFEYVKIFDEEKKKNFILLESLISTLYKKP--KSAKFKVVEKILG TPWTLPGNVAAAVHPEYTYAAFQVGDE - -ALILEEGLGR- - - KL - - LGEGTQVLKTFPG ****:*.*:* :*:*: $*$ : *** $\therefore:$ : $\quad *$

KELEWVVAQ- - - - - HPFLDRESLVINGDHVTTDAGTGCVHTAPGHGEDDYIVGQQYEL KDLVGLKYKPLFNYFYEDFKDTGFRVIPADYVTNDSGTGIVHQAPSYGEEDFNSTKAAGV KALEGLPYTPPYP - - - QALEKGYFVVLADYVSQEDGTGIVHQAPAFGAEDLETARVYGL

1FFY_2|Chain 6LDK_1|Chain 1JZQ_1|Chain

PVIS - - - -PIDDKGVFTEEGGQFEGMFYDKANKAVTDLLTEKGALLKLDFITHSYPHDW INEKKLPPSIVDDSGRMESNVPEIAGMYFKDADKVIIKKLSEEGRLLVNTQVKHSYPFCW PLLK - - - - TVDEEGK - - LLVEPFKGLYFREANRAILRDLRGRGLLFKEESYLHSYPHCW

$$
: * * *
$$$$
: *::: * *:: \text { : }
$$ 
1FFY_2|Chain 6LDK_1|Chain 1JZQ 1 Chain

1FFY_2|Chain 6LDK_1|Chain 1JZQ_1|Chain

1FFY_2|Chain 6LDK_1|Chain 1JZQ_1|Chain

1FFY_2|Chain 6LDK_1|Chain 1JZQ_1|Chain

1FFY_2|Chain 6LDK_1|Chain 1JZQ_1|Chain

1FFY_2|Chain 6LDK_1|Chain 1JZQ_1|Chain

1FFY_2|Chain 6LDK_1|Chain 1JZQ 1|Chain

1FFY_2|Chain 6LDK_1|Chain 1JZQ_1|Chain

1FFY_2|Chain 6LDK_1|Chain 1JZQ_1|Chain
VWGVPLPVFYAENGEIIMTKETVNHVADLFAEHGSNIWFEREAKDLLPEGFTHP-GSPNG YWGTPIPLWVSDDFEEMVCVGSIQELR---ELSGRD- -DITDIHRESIDSITIPSKKGKG YWGTPLPIWVCQACGKEEAIGSFQELK -- -ARATKPLPEPFDPHRPYVDQVELACA-CGG **.*:*: : : $\therefore \therefore$ : :

TFTKETDIMDVWFDSGSSHRGVLE - . - - TRPELSFPADMYLEGSDOYRGWFNSSITTS QLKRIEEVFDCWFESGSMPYASKHYPFENEKKFLDAFPANFISEGLDQTRGWFYTLTVLG TMRRVPYVIDVWYDSGAMPFASLHYPFEHEEVFRESFPADFIAEGIDQTRGWFNSLHQLG

VATRGVSPYKFLLSHGFVMDGEGKKMSKSLGNVIVPDQVVKQKGADIARLWVSSTDYLATHLFKTAPYQNVIVTGIVLAADGKKMSKRLKNYPDPTLVLEKYGADALRLYLINSPVLRA VMLFGSIAFKNVICHGLILDEKGQKMSKSKGNVVDPWDIIRKFGADALRWYIYVSAPPEA $\therefore:: *::$ : $*$ : **** $* * \quad: \therefore: * * * *::$ :

-DVRISDEILKQTSDD-YRKIRNTLRFMLGNINDFNPDTD-----SIPESELLEVDRYLL 682 ETLKFKEEGVKEIVSSVLLPWYNSYKFLKDAADLFKKDNGKDFVYDNSLHSTNVMDRWLL 704 -DRRFGPNLVRETVRDYFLTLWNVYSFFVTYANLDRPD-- - -LKNPPPPEKRPEMDRWLL 681 $\therefore:::$ : * * : : * $*$ :**:**

NRLREFTASTINNYENFDYLNIYQEVQNFINVELSNFYLDYGKDILY-IEQRDSHIRRSM 741 ASIQSLIKFIHEEMTGYRLYTVVPRLLH-FIDDLTNWYIRFNRRRIKGYASEDVEDTQKG 763 ARMQDLIQRVTEALEAYDPTTSARALRDFVVEDLSQWYVRRNRRRF--WKNEDALDREAA 739 $\therefore:$ :

QTVLYQILVDMTKLLAPILVHTAEEVWSHTP - - - - - - - - HVKEESVHLADMP LNTLVEALLTLSRAMAPFTPYLADGIYQRIKVYFKQEDLEKIAINPKNVDLRSVHFLSYP YATLYEALVLVATLAAPFTPFLAEVLWQNLVRSVRLEA-- - - - - - - KESVHLADWP $.^{*}: *::: \quad * *:$. *: : :.. .***: .*

KVVEVDQALLDKWRTFMNLRDDVNRALETARNEKVIGKSLEAKVTIASNDKFNASEFLTS 845 SVR - - -QELFDEKIEVA--VARMQKVIDMARNIR- - - - - EKKM- - - - - - - - - 856

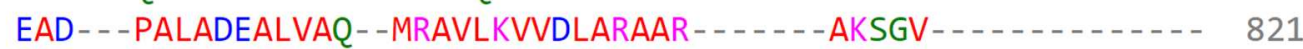
. $* * *:::: * *: \quad$ : $\quad *$

FDALHQLFIVSQVKVVDKLDDQATAYEHGDIVIEHADGEKCERCWNYSEDLGAVDELTHL 905 856 821

Fig. S8. Sequence alignment of ${ }^{S a}$ IleRS $\left(1 \mathrm{FFY} \_2{ }^{24}\right),{ }^{C a}$ IleRS $(6 \mathrm{LDK})^{25},{ }^{T}$ IleRS $(1 \mathrm{JZS})^{26}$. The amino acids of the catalytic domain of ${ }^{S a}$ IleRS are marked by a blue line. The percentage of conservation between ${ }^{S a}$ IleRS (1FFY_2), ${ }^{C a}$ IleRS (6LDK) is 35\%. The sequence of the catalytic domain is indicated by a blue line on top. 
S9. Computation of tunnel length and bottleneck radius of ${ }^{\mathrm{Ca}} \mathrm{IleRS}$ : MRC complex

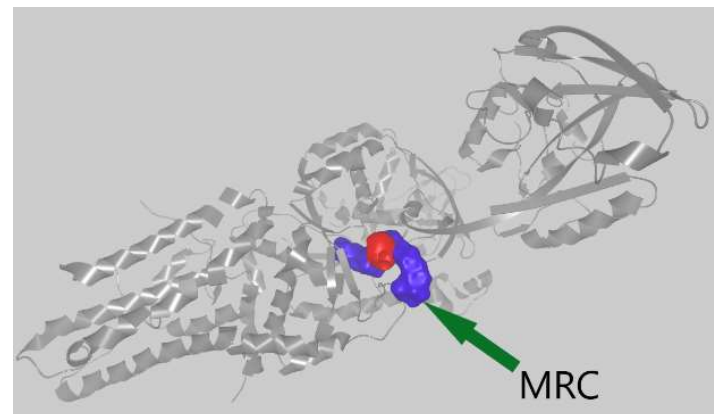

(a)

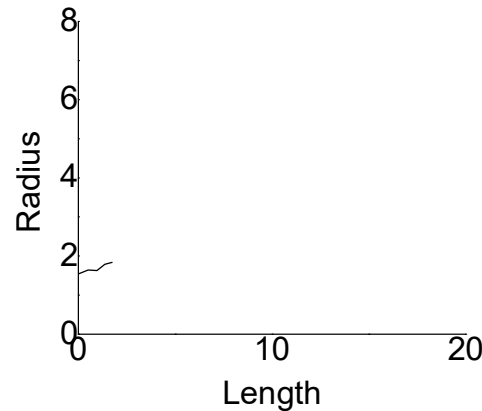

(b)

Fig. S9. The tunnel spanning the catalytic active site (red-coloured) and the plot of bottleneck radius versus length of the tunnel for ${ }^{C a}$ IleRS: MRC complex from the 1 ns simulation of the complex.

S10. Comparison of the structures of ${ }^{C a}$ IleRS: MRC complex and ${ }^{S a}$ IleRS: tRNA: MRC complex.

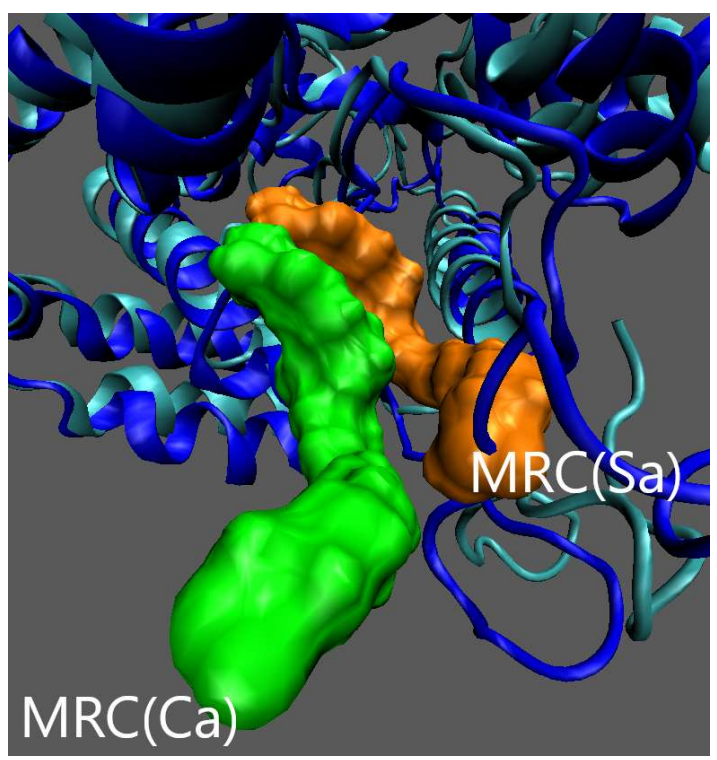

Fig. S10. Superimposed structures of ${ }^{C a}$ IleRS: MRC complex and ${ }^{S a}$ IleRS: tRNA: MRC complex. 
S11. Superimposed structures of simulated ${ }^{S a}$ IleRS:MRC:tRNA complex and ${ }^{C a}$ IleRS:MRC complex and comparison of the proximities of (a) Thr599 (in ${ }^{\mathrm{Ca}}$ IleRS) with MRC and His585 (in ${ }^{\mathrm{Sa}}$ IleRS) with MRC and (b) Ile601 (in ${ }^{C a}$ IleRS) with MRC and Phe587 (in ${ }^{S a}$ IleRS) with MRC.

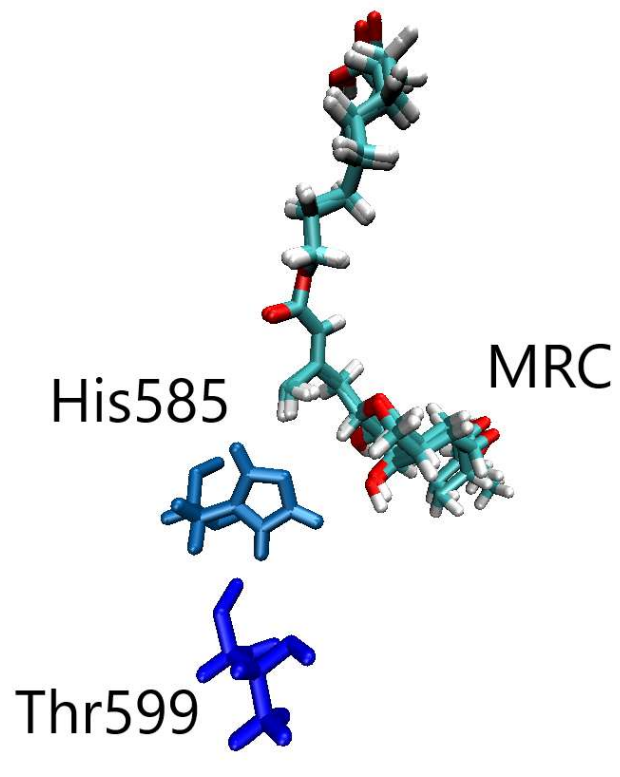

(a)

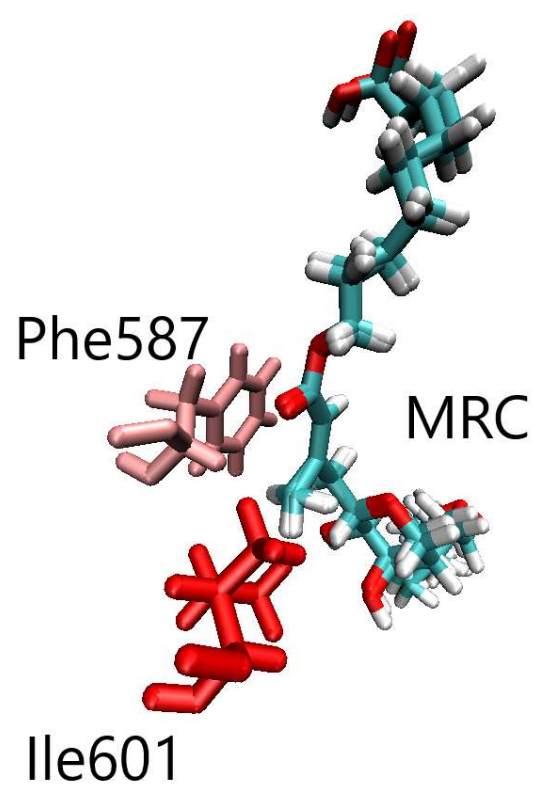

(b)

Fig. S11. Comparison of the proximities of (a) Thr599 (in ${ }^{\mathrm{Ca}} \mathrm{IleRS}$ ) with MRC and His585 (in ${ }^{S a}$ IleRS) with MRC and (b) Ile601 (in ${ }^{\mathrm{Ca}} \mathrm{IleRS}$ ) with MRC and Phe587 (in ${ }^{S a} \mathrm{IleRS}$ ) with MRC from the superimposed structures of simulated ${ }^{S a}$ IleRS:MRC:tRNA complex and ${ }^{C a}$ IleRS:MRC complex.

S12. Comparison of the structures of residue Val602 and mutated Phe602 in ${ }^{C a}$ IleRS:MRC complex.

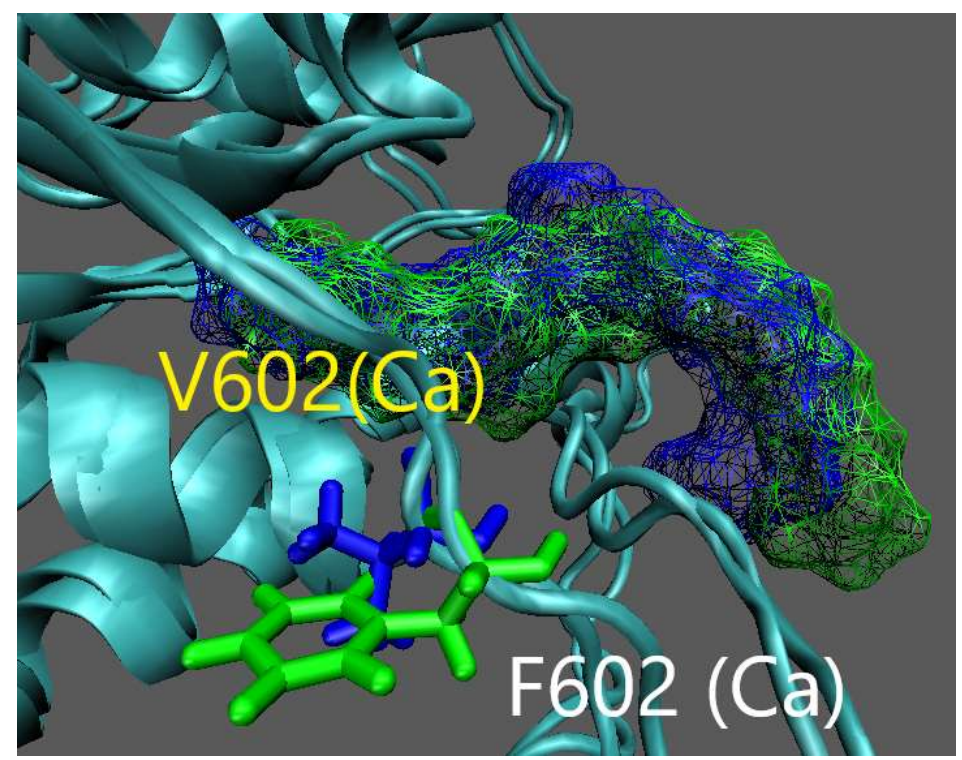

Fig. S12. Superimposed structures of residue Val602 and mutated Phe602 in ${ }^{\mathrm{Ca}}$ IleRS: MRC complex. 
S13. Comparison of the orientation and proximity of the HYGH loop and KMSKR loop with mupirocin in ${ }^{C a}$ IleRS: MRC complex. The orientation and proximity of the HMGH loop and KMSKS loop with mupirocin (represented by a line in magenta colour and marked by arrow) in ${ }^{S a}$ IleRS: tRNA: MRC complex is also compared.

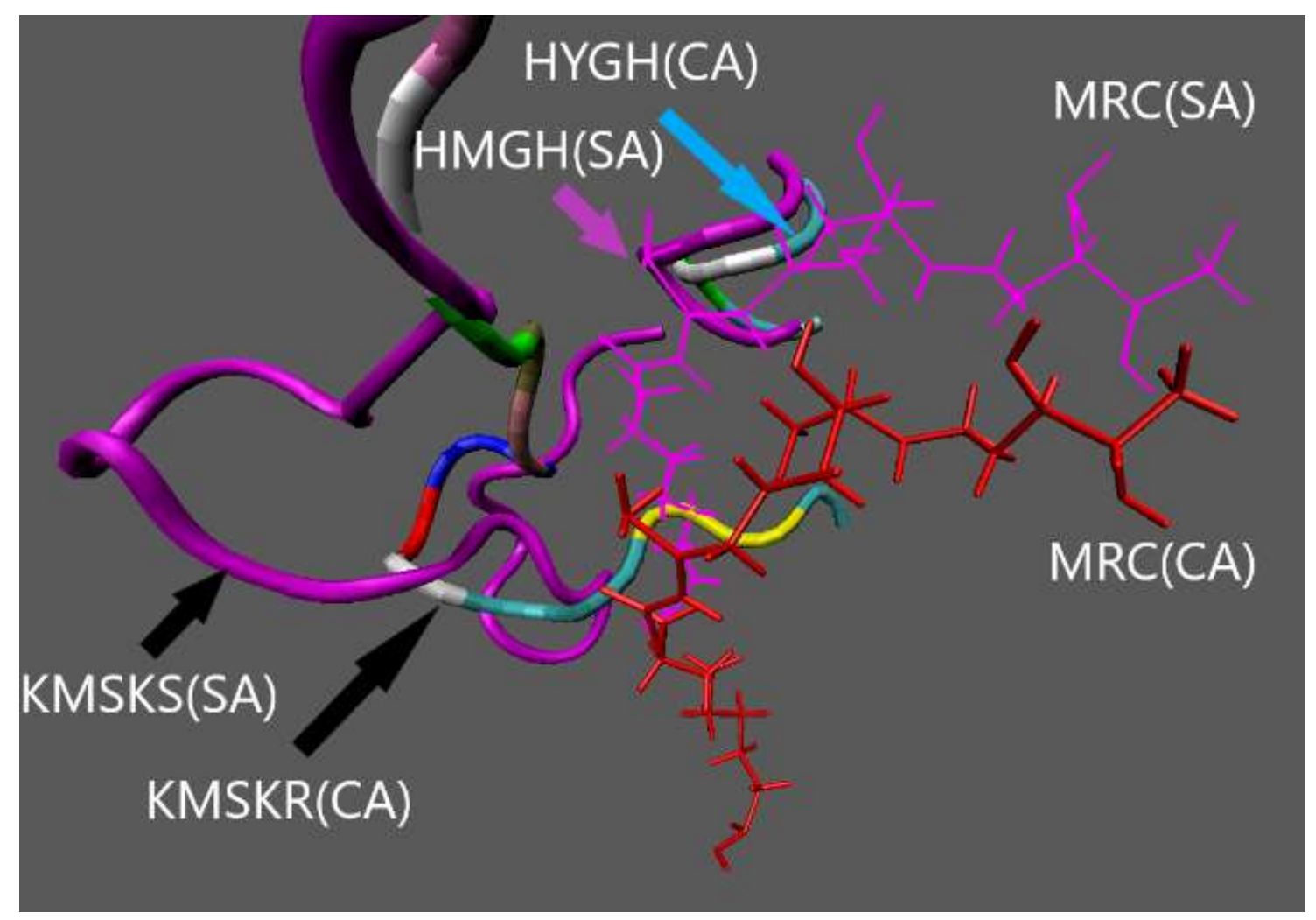

Fig. S13. Comparison of the orientation and proximity of the HYGH loop (with colours of different residues and marked by the blue arrow) and KMSKR loop (with colours of different residues and marked by arrow) with mupirocin (represented by licorice style in red colour) in ${ }^{C a} \mathrm{IleRS}$ : MRC complex. The comparison is also made with the orientation and proximity of the HMGH loop (with magenta colour and marked by magenta arrow) and KMSKS loop (with magenta colour and marked by magenta arrow) with mupirocin (represented by a line in magenta colour and marked by arrow) in ${ }^{S a}$ IleRS: tRNA: MRC complex. 